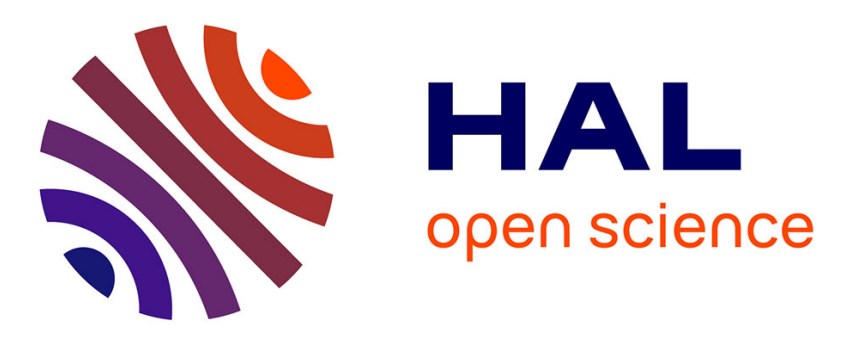

\title{
Enhanced Solid-State NMR Correlation Spectroscopy of Quadrupolar Nuclei Using Dynamic Nuclear Polarization
}

Daniel Lee, Hiroki Takahashi, Aany Thankamony, Jean-Philippe Dacquin, Michel Bardet, Olivier Lafon, Gaël de Paëpe

\section{To cite this version:}

Daniel Lee, Hiroki Takahashi, Aany Thankamony, Jean-Philippe Dacquin, Michel Bardet, et al.. Enhanced Solid-State NMR Correlation Spectroscopy of Quadrupolar Nuclei Using Dynamic Nuclear Polarization. Journal of the American Chemical Society, 2012, 134 (45), pp.18491-18494. 10.1021/ja307755t . hal-02043244

\section{HAL Id: hal-02043244 \\ https://hal.science/hal-02043244}

Submitted on 29 Sep 2021

HAL is a multi-disciplinary open access archive for the deposit and dissemination of scientific research documents, whether they are published or not. The documents may come from teaching and research institutions in France or abroad, or from public or private research centers.
L'archive ouverte pluridisciplinaire HAL, est destinée au dépôt et à la diffusion de documents scientifiques de niveau recherche, publiés ou non, émanant des établissements d'enseignement et de recherche français ou étrangers, des laboratoires publics ou privés. 


\title{
Enhanced solid-state NMR correlation spectroscopy of quadrupolar nuclei using dynamic nuclear polarization
}

\author{
Daniel Lee ${ }^{\dagger}$, Hiroki Takahashi ${ }^{\dagger}$, Aany S. L. Thankamony ${ }^{\S}$, Jean-Philippe Dacquin ${ }^{\S}$, Michel \\ Bardet $^{\dagger}$, Olivier Lafon ${ }^{\S}$, Gaël De Paëpe*†. \\ ${ }^{\dagger}$ Laboratoire de Chimie Inorganique et Biologique (SCIB), UMR-E 3 CEA / UJF-Grenoble 1, Institut Nanosciences et \\ Cryogénie (INAC), F-38054 Grenoble, France. \\ §Univ. Lille Nord de France, 59000 Lille, CNRS UMR 8181, Unité de Catalyse et de Chimie du Solide (UCCS), Univ. \\ de Lille 1, Bât. C 7 , F-59652, Villeneuve d'Ascq, France \\ dynamic nuclear polarization, solid-state nuclear magnetic resonance, magic angle spinning, dipolar recoupling, \\ quadrupolar nuclei, polarization transfer, structure determination, mesoporous alumina.
}

Supporting Information Placeholder

\begin{abstract}
Owing to a true sensitivity enhancement, corresponding to 4 to 5 orders of magnitude of time-savings, for a solid-state NMR experiment performed under dynamic nuclear polarization conditions compared to a conventional solid-state NMR experiment, it is shown that it is possible to record interface-selective ${ }^{27} \mathrm{Al}-{ }^{27} \mathrm{Al}$ two-dimensional dipolar correlation spectra on mesoporous alumina, an advanced material with potential industrial applications. The low efficiency of cross-polarization and also of dipolar recoupling for quadrupolar nuclei has been completely negated using this technique. The important presence of penta-coordinated aluminium has not only been observed, but its role in bridging interfacial tetra- and hexa-coordinated aluminium has been determined. Such structural information, collected at low temperature $(\sim 103$ $\mathrm{K}$ ) and 9.4 Tesla with the use of dynamic nuclear polarization, would have been impossible to obtain under standard conditions, even using a higher magnetic field. However, here it is demonstrated that this information can be obtained in only 4 hours. This work clearly opens a new avenue for the application of solid-state NMR to quadrupolar nuclei and notably the atomic-scale structure determination of catalysis materials, such as mesoporous alumina.
\end{abstract}

Porous materials are a cornerstone of the chemical industry since they can be employed in heterogeneous catalysis, separation processes, sensors, photonics, biomaterials etc. However, their rational design is currently limited by the lack of applicable atomic-resolution characterization methods. Nuclear magnetic resonance (NMR) spectroscopy presents itself as a technique that is able to provide detailed information on the atomic-level structure and dynamics of these heterogeneous and often disordered systems. Unfortunately, the intrinsic insensitivity of NMR impedes the examination of interfaces; these regions being crucial to the properties of porous materials.
Owing to the pioneering work of Griffin and co-workers, ${ }^{1}$ recent developments in the combination of solid-state NMR (SSNMR) under Magic-Angle Spinning (MAS) with dynamic nuclear polarization (DNP) at high magnetic field have facilitated the acquisition of high-resolution NMR spectra with greatly enhanced signal-to-noise ratios $(\mathrm{S} / \mathrm{N}){ }^{2}$ This heralds the imagination and implementation of previously unrealistic experiments. In this work, one such experiment is performed on technologically and catalytically important mesoporous alumina $\left(\mathrm{Al}_{2} \mathrm{O}_{3}\right)^{3}{ }^{3}$ This thermally stable $\left(800^{\circ} \mathrm{C}\right)$ and high surface area material (typically 200-400 $\mathrm{m}^{2} \mathrm{~g}^{-1}$ ) possessing ordered mesopores (Figure 1 (a)) has a high concentration of research interests due to its catalytic significance in the production of biodiesel, ${ }^{4}$ along with many other applications. ${ }^{5}$ Utilizing 'green' and renewable fuels is forever becoming increasingly important and the understanding of mesoporous alumina and its specific role in the catalysed biodiesel production process will lead to improved manufacture methods and therefore increased system viability.

A particular obstacle when knowledge of the local environment of ${ }^{27} \mathrm{Al}$ nuclei is desired is that conventional SSNMR radio-frequency (RF) irradiation sequences return very low efficiencies for quadrupolar nuclei. However, in spite of the difficulties arising from the quadrupolar nature of ${ }^{27} \mathrm{Al}$, herein it is shown that DNP-enhanced SSNMR can be used to perform sophisticated yet fast, interface-selective characterization measurements. Previous studies have shown that when protons are only located at the surface/interface of much larger systems then cross-polarization (CP) from protons to the nuclei of interest can result in a surface/interface-selective technique. ${ }^{6}$ This technique has been combined with DNP to study surfaces with greatly improved sensitivity ${ }^{7}$ and also $\gamma$-alumina where $\mathrm{CP}$ is very inefficient. ${ }^{8}$

In this work the DNP is dominated by the cross-effect $(\mathrm{CE})^{9}$ between two unpaired electrons in a biradical 
(TOTAPOL ${ }^{10}$ ) and protons in a glass-forming solvent matrix or protons at the interface in the mesoporous alumina. The experiments were performed on a Bruker BioSpin solid-state DNP-NMR spectrometer." ${ }^{11}$ This spectrometer is equipped with a gyrotron and connecting transmission line, able to provide microwave $(\mu \mathrm{w})$ irradiation at $263 \mathrm{GHz}$ and approximately $5 \mathrm{~W}$ at the sample, in combination with an AVANCE ${ }^{\mathrm{TM}}$ III $400 \mathrm{MHz}$ wide-bore NMR system and a low-temperature MAS probe capable of achieving spinning rates for $3.2 \mathrm{~mm}$ rotors of up to $17 \mathrm{kHz}$ at sample temperatures of approximately $100 \mathrm{~K}$.

The 'DNP-ready' sample was prepared by impregnation with an excess of solution..$^{12}$ The mesoporous alumina was first heated to $408 \mathrm{~K}$ for 6 hours to ensure that the sample, including the pores, was dry. $46 \mu \mathrm{L}$ of $20 \mathrm{mM}$ TOTAPOL in a glass-forming mixture of $\left[{ }^{2} \mathrm{H}_{6}\right]$-DMSO, $\mathrm{H}_{2} \mathrm{O}$ and ${ }^{2} \mathrm{H}_{2} \mathrm{O}(78$ $\%, 8 \%, 14 \%$ according to weight, respectively) was added to cover $28 \mathrm{mg}$ of the mesoporous alumina powder. This mixture was stirred vigorously and then left to rest at room temperature for 24 hours to allow time for the impregnation of the pores by the DNP chemical matrix. A repetition of centrifuging the mixture followed by pipetting any supernatant was employed to remove the excess liquid. The remaining wet powder was added to a thin-walled zirconia $3.2 \mathrm{~mm}$ MAS rotor. It must be stated that, unlike other systems, since it is not necessary to dissolve/disperse ${ }^{1}$ the impregnated mesoporous alumina in a DNP matrix then these systems benefit from the ability to use the maximum amount of the sample of interest possible without compromising the DNP-enhancement.

(a)

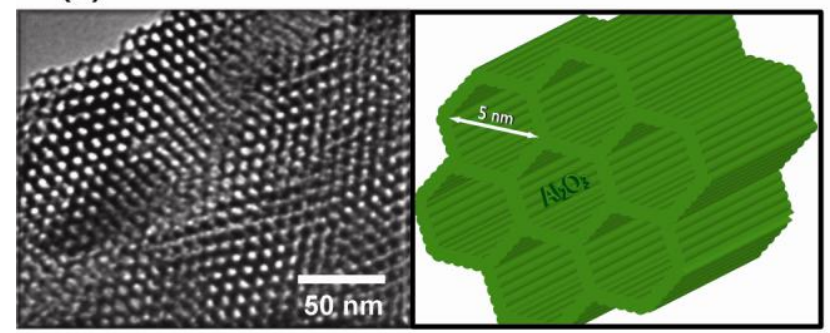

(b)

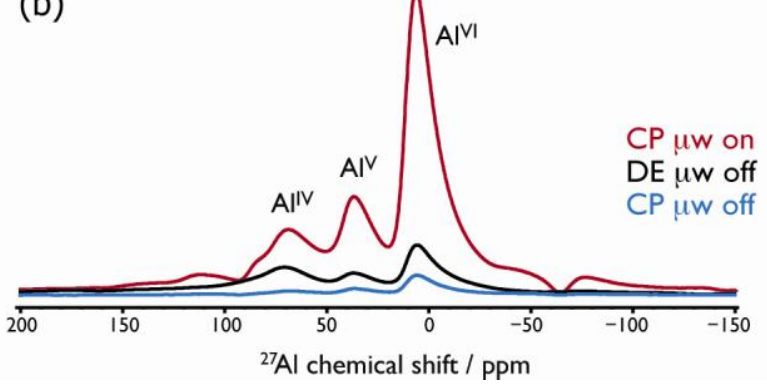

Figure 1. (a) TEM micrograph (left) and associated schematic (right) showing the presence of ordered hexagonal mesopores throughout the alumina sample studied in this work. (b) ${ }^{27} \mathrm{Al}$ NMR spectra of this sample recorded with a MAS rate of $8 \mathrm{kHz}$ and a sample temperature of $\sim 103 \mathrm{~K}$.

The NMR signal enhancement as a result of DNP in this system can be seen in Figure 1 (b). With $\mu$ w irradiation the
CE enhances the polarization of protons within the sample. This polarization is then transferred to the central transition (CT) of the ${ }^{27} \mathrm{Al}$ nuclei via low power $\mathrm{CP}$ under MAS $(\mathrm{CPMAS})^{13}$ and the resulting spectrum is recorded (red line). The experiment is repeated under the same conditions except without the $\mu \mathrm{w}$ irradiation (blue line). Here, the enhancement, $\varepsilon$, is measured simply by comparing the intensities of the spectral peaks resulting from the CPMAS experiments with and without $\mu \mathrm{w}$ irradiation. For this system $\varepsilon$ was 15 . This was the same for each of the ${ }^{27} \mathrm{Al}$ peaks. For comparison, the direct excitation under MAS (DEMAS) spectrum of ${ }^{27} \mathrm{Al}$ is also shown in Figure 1 (b) (black line). As an aside, a similar DNP-ready sample that used an impregnation time of only 1 hour was also tested. For this sample $\varepsilon$ was 11. Thus, it appears that longer impregnation times are necessary to facilitate the penetration of the TOTAPOL solution into the pores.

In the spectra of Figure 1 (b) three discrete ${ }^{27} \mathrm{Al}$ peaks are observed. These correspond to hexa-coordinated aluminium, $\mathrm{Al}^{\mathrm{VI}}$, with a chemical shift $\delta\left({ }^{27} \mathrm{Al}\right)=5 \mathrm{ppm}$, penta-coordinated aluminium, $\mathrm{Al}^{\mathrm{V}},\left(\delta\left({ }^{27} \mathrm{Al}\right)=36 \mathrm{ppm}\right)$ and tetra-coordinated aluminium, $\mathrm{Al}^{\mathrm{IV}},\left(\delta\left({ }^{27} \mathrm{Al}\right)=70 \mathrm{ppm}\right)$ sites. Contrary to the literature, ${ }^{8}$ the substantial presence of $\mathrm{Al}^{\mathrm{V}}$ resonances observed here indicates that the water-based DNP matrix does not significantly coordinate to these sites and thus this solution is applicable for the study of $\mathrm{Al}^{\mathrm{V}}$ sites using DNP-enhanced SSNMR measurements of similar systems. Furthermore, this abundance of $\mathrm{Al}^{\mathrm{V}}$ sites at the interface demonstrates the industrial potential for this mesoporous alumina. Not only does this system exhibit greater surface area than the more commonly employed $\gamma$-alumina but it appears that these $\mathrm{Al}^{\mathrm{V}}$ sites, which can act as binding sites for the active phase, ${ }^{14}$ are much more plentiful.

Not only does the impregnation solution provide the biradical necessary for DNP and the glass-forming solvents to best utilize this biradical, but this matrix also provides extra protons that enhance the $\mathrm{CP}$ signal, irrespective of DNP. Furthermore, the requirement of low temperatures for efficient DNP has the added advantage of increasing nuclear relaxation times, with the usually fast relaxation of quadrupolar nuclei during spin-lock periods limiting the success of $\mathrm{CP}$ for measurements at room temperature. The combination of low temperatures and the addition of these extra, useful protons means that the time-savings using this system for DNP-enhanced CPMAS experiments, when compared to ubiquitous SSNMR systems, cannot be predicted by simply allowing for the DNP enhancement $(\varepsilon)$, the low-temperature gain (Boltzman factor and reduced thermal noise) whilst taking into account sample 'bleaching ${ }^{\prime}$ and the change in relaxation times due to the paramagnetic effects of the added biradical. The only true comparison in this (and similar) system(s) is to record spectra under specific DNP conditions and under normal SSNMR conditions and compare the S/N per unit time. ${ }^{16}$ Note that resolution is also an extremely important factor and apparent linewidths should also be taken into account. However, in this system the linewidths are dominated by disorder and there are negligible changes between the two experimental conditions. A similar CPMAS experiment was recorded under conventional SSNMR conditions (9.4 T, 298 
$\mathrm{K}, 8 \mathrm{kHz}$ MAS on a $3.2 \mathrm{~mm}$ rotor; not shown). Comparing the $\mathrm{S} / \mathrm{N}$ per unit time between the DNP-enhanced CPMAS spectrum and the conventional CPMAS spectrum showed that using DNP for this system resulted in a true enhancement factor of 184 (time-saving factor of $\sim 34000$ ). This demonstrates the considerable advantage of using DNP in the study of this system.

A comparison of the NMR signal intensity for each aluminium coordination state between the DNP-enhanced CPMAS experiment and the DEMAS experiment can only give a very crude indication of the influence of protons on each state. The ${ }^{27} \mathrm{Al}^{\mathrm{IV}}$ peak is 2.4 times larger in the DNPenhanced CPMAS spectrum, the ${ }^{27} \mathrm{Al}^{\mathrm{V}}$ peak is 4.6 times larger and the ${ }^{27} \mathrm{Al}^{\mathrm{VI}}$ peak is 6.1 times larger. Note that the linewidths (and thus the apparent transverse dephasing times, $T_{2}{ }^{*}$ ) do not vary between the two spectra and, accordingly, proton couplings are not the limiting factor of these linewidths. A more complete analysis of the relative influence of protons can be performed using measurements of the time constant associated with the homogeneous contribution to the transverse dephasing which cannot be refocused with a $\pi$-pulse, $T_{2}^{\prime}$. $T_{2}^{\prime}$ values were measured using a pseudo-two-dimensional spin-echo experiment with varied, rotor-synchronized echo times, the pulse sequence for which is given in Figure S1. Figure 2 illustrates such measurements for each of the aluminium peaks in the mesoporous alumina sample, both with and without heteronuclear decoupling. This is used to demonstrate the effect protons have on each $T_{2}^{\prime}$, and thus on each aluminium site. Note that the employed heteronuclear decoupling was deemed sufficient in that an increase in power did not affect the value for $T_{2}^{\prime}$ (this was observed for optimized SPINAL- $64^{17}$ decoupling with powers above $90 \mathrm{kHz}$; data not shown). The ratio of the $T_{2}$ ' value measured with decoupling to the value measured without decoupling was 2.3 for $\mathrm{Al}^{\mathrm{IV}}$, 2.9 for $\mathrm{Al}^{\mathrm{V}}$ and 3.2 for $\mathrm{Al}^{\mathrm{VI}}$. This is in agreement with the relative intensities between the CPMAS and DEMAS spectra. These corroborating observations indicate that the interfacial $\mathrm{Al}^{\mathrm{VI}}$ sites are more heavily influenced by protons than the interfacial $\mathrm{Al}^{\mathrm{V}}$ sites which, in turn, are more heavily influenced by protons than the interfacial $\mathrm{Al}^{\mathrm{IV}}$ sites. This is consistent with an increase in the number of hydroxo or aqua groups for an increasing coordination number.

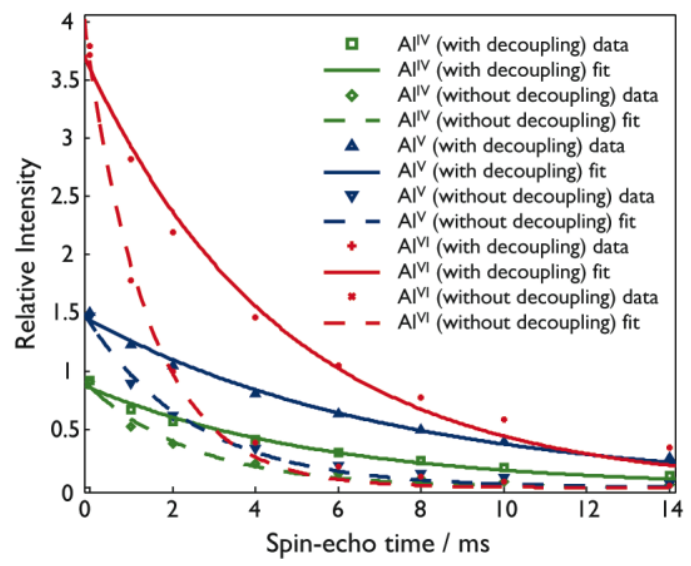

Figure 2. Normalized intensity decay for each ${ }^{27} \mathrm{Al}$ peak with and without $100 \mathrm{kHz}$ of SPINAL-64 heteronuclear decoupling during a spin-echo period as a function of the (rotor-synchronized) spin-echo time, used to determine $T_{2}$ '. This DNPenhanced experiment was performed with a MAS rate of 8 $\mathrm{kHz}$, a sample temperature of $\sim 103 \mathrm{~K}$ and the pulse sequence shown in Figure S1.

The lack of change in $T_{2}{ }^{*}$ for each spectral peak between the CPMAS and DEMAS experiments noted above could indicate that heteronuclear decoupling is not necessary. Nonetheless, as can clearly be seen from Figure 2, protons have a significant impact on the spin dynamics of ${ }^{27} \mathrm{Al} \mathrm{nu-}$ clei. Moreover, in this system the large influence of protons is not just limited to experiments that begin with a $\mathrm{CP}$ step. However, this realization is beyond the scope of this work but will be discussed in detail in a future publication.

Figure 2 illustrates the importance of the heteronuclear decoupling during the spin-echo period; these periods (and thus $T_{2}^{\prime}$ values) being vital in most solid-state NMR experiments involving $J$-couplings..$^{18} \mathrm{~A}$ further demonstration of its importance is given in Figure $\mathrm{S}_{2}$ where the spectral intensities of each ${ }^{27} \mathrm{Al}$ coordination state after $\mathrm{CP}$ and then a spin-echo period of total duration $4 \mathrm{~ms}$ are compared with and without ${ }^{1} \mathrm{H}$ decoupling. Globally the intensities without decoupling are reduced by $60 \%$ compared to those with decoupling. $T_{2}^{\prime}$ values are also important in solid-state NMR experiments involving dipolar couplings (REDOR-type experiments etc. ${ }^{19}$ ). Furthermore, other dipolar recoupling sequences will experience magnetization decay times on the order of $T_{2}^{\prime}$ which can also be heavily affected by proton couplings. Thus, heteronuclear decoupling during these sequences can also be highly beneficial, as long as interference effects are avoided.

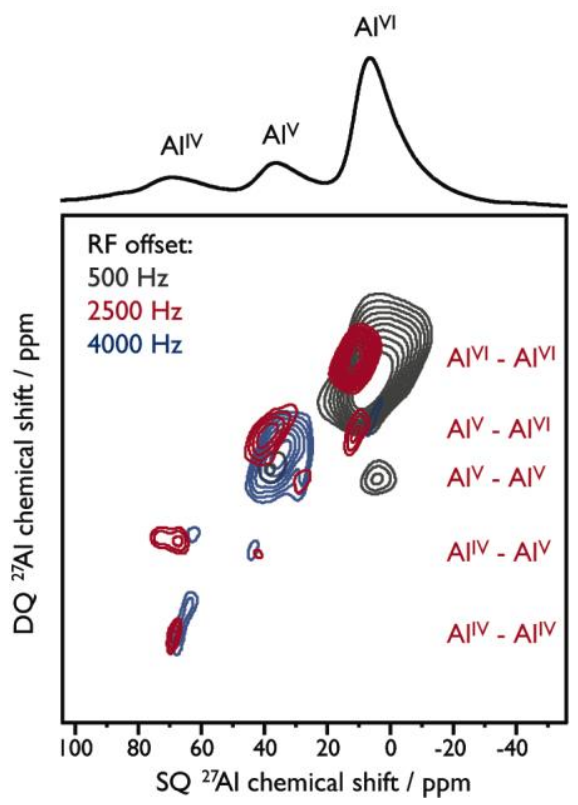

Figure 3. DNP-enhanced, interface-selective DQ-SQ ${ }^{27} \mathrm{Al}$ homonuclear dipolar correlation spectra of the mesoporous alumina recorded with a MAS rate of $8 \mathrm{kHz}$, a sample temperature of $\sim 103 \mathrm{~K}$ and the pulse sequence shown in Figure S1. 
Total acquisition time for each spectrum was $\sim 4$ hours. For illustrative purposes, the DNP-enhanced CPMAS spectrum (Figure $1(\mathrm{~b})$ ), with the three different aluminium peaks labeled, is also shown.

The supercycled symmetry-based dipolar recoupling sequence $\mathrm{BR} 2_{2}{ }^{1}$ has been shown to be of great utility when recording homonuclear correlation spectra. ${ }^{20}$ However, the use of this, and other, ${ }^{21}$ sequences for ${ }^{27} \mathrm{Al}$ has been limited to bulk studies by the low efficiency (approximately $5 \%$ ), which results from the intricate spin dynamics of quadrupolar nuclei in the presence of RF field and MAS. The entirety of the adapted pulse sequence used here for interface-selective, DNP-enhanced correlation spectroscopy of quadrupolar nuclei is given in Figure $\mathrm{S}$. This pulse sequence firstly transfers hyperpolarized proton magnetization to nearby half-integer spin quadrupolar nuclei via a $\mathrm{CP}$ step. Then, the sequence creates double-quantum (DQ) coherences between the CTs of dipolar-coupled quadrupolar nuclei whilst removing DQ coherence contributions involving satellite transitions of a single ${ }^{27} \mathrm{Al}$ nucleus. The spectra resulting from this sequence then give information about the spatial proximities between neighboring nuclei. The variables of this experiment can be optimized in a one-dimensional fashion (no indirect-dimension increments). Using this $1 \mathrm{D}$ experiment the importance of heteronuclear decoupling during the recoupling periods and also the whole sequence can be clearly demonstrated (Figure S2). Moreover, demanding experiments usually require careful calibrations of pulses and delays. Here, calibrations of the parameters required for maximum efficacy of the recoupling sequence could be performed on the sample of interest in less than an hour owing to the huge gain in S/N due to the DNP conditions. Under conventional SSNMR conditions these calibrations would require much longer experimental times even with model samples.

Once calibrated, the two-dimensional version of this pulse sequence was used to record the first correlation spectra of quadrupolar nuclei using DNP. Furthermore, since the sequence begins with a CP step, observed correlations would only be between interfacial ${ }^{27} \mathrm{Al}$ nuclei. Spectra resulting from the implementation of this sequence on the mesoporous alumina sample are shown in Figure 3. It has been shown that the $\mathrm{BR}_{2}{ }^{1}$ recoupling sequence has a dependency on offset ${ }^{19 a}$ so three spectra with different offsets were recorded to remove any uncertainty in the data. Possibly the most striking point is that each spectrum only took approximately four hours to acquire. Under conventional SSNMR conditions the acquisition of equivalent data would require over fifteen years for each spectrum! It can be seen that dipolar correlations between all sites are present (including same site correlations between different nuclei e.g. $\mathrm{Al}^{\mathrm{VI}}-\mathrm{Al}^{\mathrm{VI}}$ correlations) except for correlations between $\mathrm{Al}^{\mathrm{IV}}$ and $\mathrm{Al}^{\mathrm{VI}}$ sites. Therefore, at the interface of this material $\mathrm{Al}^{\mathrm{V}}$ sites connect $\mathrm{Al}^{\mathrm{IV}}$ and $\mathrm{Al}^{\mathrm{VI}}$ sites. To summarize, DNP enhancements $(\varepsilon)$ of about 15 have been observed on an industrially important sample of mesoporous alumina using a 9.4 $\mathrm{T}$ solid-state DNP-NMR spectrometer. More importantly, this translates into a true sensitivity gain per unit time of 184 when compared to equivalent room temperature measurements. Transcending the sensi- tivity limitations of cross-polarization and also dipolar recoupling for quadrupolar nuclei, the corresponding 4 to 5 orders of magnitude of time-savings allow not only the acquisition of very fast $1 \mathrm{D}$ CPMAS experiments and pseudo$2 \mathrm{D}$ experiments but also interface-selective $2 \mathrm{D}$ homonuclear dipolar correlation experiments. These $1 \mathrm{D}$ and $2 \mathrm{D}$ experiments clearly demonstrate that it is possible to probe spatial proximities involving quadrupolar nuclei with the use of DNP-enhanced SSNMR with greatly reduced timescales. Moreover, the absence of $\mathrm{Al}^{\mathrm{IV}}-\mathrm{Al}^{\mathrm{VI}}$ correlations combined with the presence of $\mathrm{Al}^{\mathrm{IV}}-\mathrm{Al}^{\mathrm{V}}$ and $\mathrm{Al}^{\mathrm{V}}-\mathrm{Al}^{\mathrm{VI}}$ correlations shows that interfacial $\mathrm{Al}^{\mathrm{V}}$ bridges interfacial $\mathrm{Al}^{\mathrm{IV}}$ and $\mathrm{Al}^{\mathrm{VI}}$ sites. This structural data, together with the results of the influence of protons on the $T_{2}$ ' of each interfacial site, can be combined with $a b$ initio calculations to help elucidate the surface structure of this amorphous material. This work is planned for a future publication.

\section{ASSOCIATED CONTENT}

Supporting Information Placeholder

Figures $\mathrm{S}_{1}$ and $\mathrm{S}_{2}$. Experimental details and pulse sequence diagrams along with spectra demonstrating the importance of heteronuclear decoupling for this study. This material is available free of charge via the Internet at http://pubs.acs.org.

\section{AUTHOR INFORMATION}

\section{Corresponding Author}

*gael.depaepe@cea.fr

\section{ACKNOWLEDGMENTS}

This work was supported by the ANR (ANRo8-CEXC-oo3-o1) and funding from the RTB. G.D.P. was supported by EU Marie Curie (PIEF-GA- 2009-237646) for part of the work. D.L. was supported by CEA-EUROTALENTS (PCOFUND-GA-20o8228664). Dr. M. Giffard is acknowledged for the synthesis of the TOTAPOL biradical. The UCCS members are grateful for funding provided by Region Nord/Pas de Calais, Europe (FEDER), CNRS, French Minister of Science, FR-3050, USTL, ENSCL, and ANR-2010-JCJC-o811-01. O. L. and A.S.L.T. acknowledge X. Lu, J. Trébosc, H. Vezin and J.-P. Amoureux for helpful discussions.

\section{REFERENCES}

(1) Hall, D.A.; Maus, D.C.; Gerfen, G.J.; Inati, S.J.; Becerra, L.R.; Dahlquist, F.W.; Griffin, R.G. Science 1997, 276, 930.

(2) Barnes, A.B.; Paëpe, G.D.; van der Wel, P.C.; Hu, K.N.; Joo, C.G.; Bajaj, V.S.; Mak-Jurkauskas, M.L.; Sirigiri, J.R.; Herzfeld, J.; Temkin, R.J.; Griffin, R.G. Appl. Magn. Reson. 2008, 34, 237.

(3) Dacquin, J.-P.; Dhainaut, J.; Duprez, D.; Royer, S. ; Lee, A.F.; Wilson, K. J. Am. Chem. Soc. 2009, 131, 12896.

(4) Wilson, K.; Lee, A.F.; Dacquin, J.-P. Book Chapter in Catalysis for Alternative Energy Generation (Springer) 2012, 263.

(5) (a) Bejenaru, N.; Lancelot, C.; Blanchard, P.; Lamonier, C.; Rouleau, L.; Payen, E.; Dumeignil, F.; Royer, S.; Chem. Mater. 2009, 21, 522. (b) Granger, P.; Parvulescu, V.I. Chem. Rev. 2011, 111, 3155 .

(6) (a) Agarwal, G.K.; Titman, J.J.; Percy, M.J.; Armes, S.P. J. Phys. Chem. B. 2003, 107, 12497. (b) Lee, D.; Balmer, J.A.; Schmid, A.; Tonnar, J.; Armes, S.P.; Titman, J.J. Langmuir 2010, 26, 15592.

(7) (a) Lock, H.; Maciel, G.E.; Johnson, C.E. J. Mater. Res. 1992, 7, 2791. (b) Lesage, A.; Lelli, M.; Gajan, D.; Caporini, M.A.; Vitzthum, 
V.; Miéville, P.; Alauzun, J.; Roussey, A.; Thieuleux, C.; Mehdi, A.; Bodenhausen, G.; Copéret, C.; Emsley, L. J. Am. Chem. Soc. 2010, 132, 15459. (c) Rossini, A.J.; Zagdoun, A.; Lelli, M.; Canivet, J.; Aguado, S.; Ouari, O.; Tordo, P.; Rosay, M.; Maas, W.E.; Copéret, C.; Farrusseng, D.; Emsley, L.; Lesage, A. Angew. Chem. Int. Ed. Engl. 2012, 51, 123.

(8) Vitzthum, V.; Miéville, P.; Carnevale, D.; Caporini, M.A.; Gajan, D.; Copéret, C.; Lelli, M.; Zagdoun, A.; Rossini, A.J.; Lesage, A.; Emsley, L.; Bodenhausen, G. Chem. Commun. 2012, 48, 1988.

(9) (a) Kessenikh, A.V.; Manenkov, A.A.; Pyatnitskii, G.I. Sov. Phys. Solid State, 1964, 6, 641. (b) Hwang, C.F.; Hill D.A. Phys. Rev. Lett. 1967, 19, 1011.

(10) Song, C.; Hu, K.N.; Joo, C.G.; Swager, T.M.; Griffin, R.G. J. Am. Chem. Soc. 2006, 128, 11385.

(11) Rosay, M.; Tometich, L.; Pawsey, S.; Bader, R.; Schauwecker, R.; Blank, M.; Borchard, P.M.; Cauffman, S.R.; Felch, K.L.; Weber, R.T.; Temkin, R.J.; Griffin, R.G.; Maas, W.E. Phys. Chem. Chem. Phys. 2010, 12, 5850.

(12) (a) Haber, J.; Block, J.H.; Delmon, B. Pure Appl. Chem. 1995, 67, 1257. (b) Lafon, O.; Rosay, M.; Aussenac, F.; Lu, X.Y.; Trebosc, J.; Cristini, O.; Kinowski, C.; Touati, N.; Vezin, H.; Amoureux, J.P. Angew. Chem. Int. Ed. 2011, 50, 8367.

(13) (a) Vega, A.J. Solid State Nucl. Magn. Reson. 1992, 1, 17. (b) Amoureux, J.-P.; Pruski, M. Mol. Phys. 2002, 100, 1595.

(14) (a) Kwak, J.H.; Hu, J.Z.; Kim, D.H.; Szanyi, J.; Peden, C.H.F. J. Catal. 2007, 251, 189. (b) Kwak, J.H.; Hu, J.; Mei, D.; Yi, C.-W.;
Kim, D.H.; Peden, C.H.F.; Allard, L.F.; Szanyi, J. Science 2009, 325, 1670.

(15) (a) Thurber, K.R.; Yau, W.-M.; Tycko, R.J. Magn. Reson. 2010, 204, 303. (b) Vitzthum, V.; Borcard, F.; Jannin, S.; Morin, M.; Mieville, P.; Caporini, M.A.; Sienkiewicz, A.; Gerber-Lemaire, S.; Bodenhausen, G. ChemPhysChem 2011, 12, 2929.

(16) Takahashi, H.; Lee, D.; Dubois, L.; Bardet, M.; Hediger, S.; De Paëpe, G. Angew. Chem. Int. Ed. DOI: 10.1002/anie.201206102.

(17) Fung, B.M.; Khitrin, A.K.; Ermolaev, K. J. Magn. Reson. 2000, 142, 97.

(18) (a) Fyfe, C.A.; Wong-Moon, K.C.; Huang, Y.; Grondey H. J. Am. Chem. Soc. 1995, 117, 10397. (b) Lesage, A.; Bardet, M.; Emsley, L. J. Am. Chem. Soc. 1999, 121, 10987. (c) Lee, D.; Struppe, J.; Elliott, D.W.; Mueller, L.J.; Titman, J.J. Phys. Chem. Chem. Phys. 2009, 11, 3547 .

(19) Gullion, T.; Schaefer, J. J. Magn. Reson. 1989, 81, 196.

(20) (a) Wang, Q.; Hu, B.; Lafon, O.; Trebosc, J.; Deng, F.; Amoureux, J.P. J. Magn. Reson. 2009, 200, 251. (b) Lafon, O.; Trebosc, J.; Hu, B.W.; De Paëpe, G.; Amoureux, J.P. Chem. Commun. 2011, 47, 6930 .

(21) (a) Eden, M.; Zhou, D.; Yu, J. Chem. Phys. Lett. 20o6, 431, 397. (b) Hansen, M.R.; Jakobsen, H.J.; Skibsted J. J. Phys. Chem. C 2008, 112, 7210 .

\section{TOC Graphic}

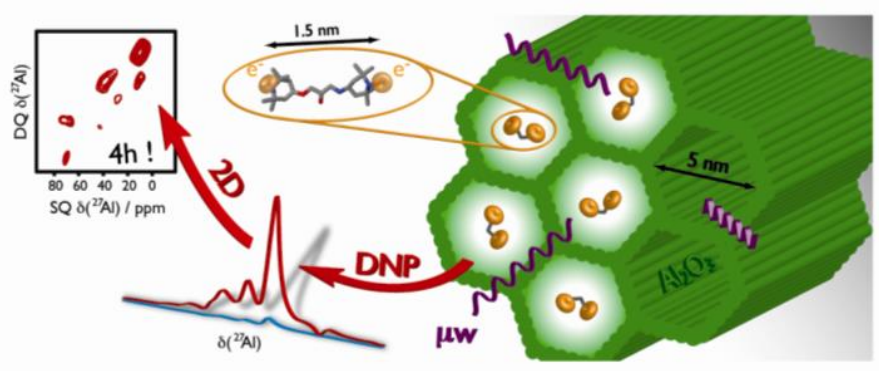

